\title{
Smartlink: Exploiting Channel Clustering Effects for Reliable Millimeter Wave Communications
}

\author{
Irmak Aykin $^{1,2}$, Berk Akgun ${ }^{1}$, and Marwan Krunz ${ }^{1,2}$ \\ ${ }^{1}$ Department of Electrical and Computer Engineering, University of Arizona, Tucson, AZ \\ ${ }^{2}$ FreeFall Aerospace Inc., Tucson, AZ \\ \{aykin, berkakgun, krunz\}@email.arizona.edu
}

\begin{abstract}
Millimeter wave $(\mathrm{mmW})$ communications have recently attracted considerable attention as a key element of nextgeneration (5G) wireless systems. Despite significant efforts in this domain, establishing and maintaining directional $\mathrm{mmW}$ links in a dynamic environment are still quite challenging, largely due to the search-time overhead of beam scanning, and the vulnerability of directional links to beam misalignment, blockage, and outages. In this paper, we propose SmartLink, a protocol that exploits the multi-cluster scattering phenomenon at $\mathrm{mmW}$ frequencies to establish a multi-directional link between a base station and a user. By exploiting multiple clusters, SmartLink enables fast initial access and link maintenance, along with sustained throughput. A search algorithm called multi-lobe beam search (MLBS) is used to discover multiple channel clusters by probing several directions simultaneously using carefully designed multilobe beam patterns. MLBS reduces the search time from linear to logarithmic with respect to the number of directions. We provide detailed analysis of the false alarm and misdetection probabilities for the designed beam patterns. Following cluster discovery, SmartLink divides antennas into sub-arrays to generate the optimal multi-lobe pattern with respect to cluster powers and blockage probabilities. Finally, extensive trace-driven simulations at $29 \mathbf{G H z}$ frequency using phased-array antennas verify the efficiency of SmartLink.
\end{abstract}

Index Terms-Millimeter wave; initial access; analog beamforming; blockage; trace-driven simulations.

\section{INTRODUCTION}

Millimeter wave $(\mathrm{mmW})$ bands represent the new frontiers for wireless communications due to the massive spectrum available at these frequencies (e.g., about $3 \mathrm{GHz}$ in the $28 \mathrm{GHz}$ and $39 \mathrm{GHz}$ bands, and $5-9 \mathrm{GHz}$ in the $60 \mathrm{GHz}$ band) [1] Next-generation wireless technologies such as $5 \mathrm{G}$ and WiGig are expected to rely on $\mathrm{mmW}$ bands to satisfy the growing demand for mobile data [2], [3]. However, operating wireless devices over mmW bands faces many challenges, including significant path loss (compared to sub-6 GHz bands [4]), inability to penetrate walls, and vulnerability to blockage [1]. On the other hand, the shorter wavelengths allow large number of antenna elements to be integrated onto a small form-factor radio. With proper analog and/or digital processing of signals fed into these antenna elements, transmissions can be beamed along specific directions. The severe signal attenuation at $\mathrm{mmW}$ frequencies can then be compensated for by the high beamforming gain, providing sufficient link margin [5].

There are several ways to apply beamforming at $\mathrm{mmW}$ frequencies. Analog beamforming can be used with only one RF chain and multiple phase shifters that feed an antenna array and steer the beam. In contrast, digital beamforming relies on multiple RF chains (as many as the number of antenna elements) and multiplies a particular precoding matrix by the modulated baseband signals of various RF chains. Despite its superior performance, pure digital beamforming is less favored due to its higher complexity and energy consumption. Accordingly, hybrid beamforming in which the signal processing is divided between the analog and digital domains, has been proposed [6]. Still, the high cost and power consumption of having multiple RF chains force current $\mathrm{mmW}$ systems to rely heavily on analog beamforming.

Establishing and maintaining network connectivity are key challenges in directional mmW systems [7], [8]. Due to fewer reflections in $\mathrm{mmW}$ spectrum, the channel matrix between the base station (BS) and the user equipment (UE) is sparse [5], [9]. More specifically, transmitted signals reach the receiver in a few (typically less than 5) distinct angular clusters, depending on the environment. Identifying the channel clusters to be used for the communications takes considerable amount of time. Furthermore, mmW signals have significantly lower diffraction ability compared to sub- $6 \mathrm{GHz}$ bands, making them very vulnerable to blockage [10], [11].

In this paper, we propose an efficient and reliable communication protocol for $\mathrm{mmW}$ systems called SmartLink. SmartLink utilizes multiple clusters between the BS and the UE to provide an effective mechanism for maintaining communications in case of blockage. It uses a unique initial access (IA) scheme called multi-lobe beam search (MLBS), which relies on shortest-depth decision trees. MLBS relies on carefully designed beam patterns with multiple lobes to simultaneously discover multiple channel clusters. With MLBS, the search time can be reduced from linear to logarithmic with respect to the total number of scanned directions. The creation of multiple main lobes naturally reduces the beamforming gain, with negative impact on the false alarm and misdetection probabilities. We provide detailed analyses of both probabilities. Here, misdetection is defined as the inability to discover the UE due to low received power. False alarm refers to the event when a channel cluster is aligned with an undesired side lobe of the transmit (Tx) and/or the receive ( $\mathrm{Rx}$ ) antenna array (resulting in sub-optimal communication). Depending on the discovered channel clusters and their relative gains, we virtually divide the $\mathrm{Tx}$ and the $\mathrm{Rx}$ antenna arrays into subarrays, each generating a beam towards one of the inferred 
channel clusters, so as to maximize the average data rate in a blockage-prone environment.

The main contributions of this paper are as follows:

- We introduce a logarithmic-time search scheme called MLBS for the discovery of multiple channel clusters in a directional $\mathrm{mmW}$ system. MLBS relies on analog beamforming at both the BS and the UE.

- We provide detailed analyses of the misdetection and false alarm probabilities under MLBS.

- We provide a technique for splitting the antenna array into sub-arrays to generate an optimal multi-lobe pattern. This splitting depends on the discovered channel clusters, their relative strengths, and their blockage probabilities.

- We verify the efficiency of SmartLink in terms of the reduction in the search time and the increase in the data rate by conducting experimental channel measurements at $29 \mathrm{GHz}$ frequency using $4 \times 4$ uniform planar arrays (UPAs) and running trace-driven simulations using these channel measurements. With a proper number of main lobes of the antenna pattern, SmartLink reduces the search latency by up to $65 \%$ compared to $802.11 \mathrm{ad}$ and $5 \mathrm{G}$ NR beam scanning approaches. Our results also show that utilizing multiple clusters provides an efficient mechanism against blockages, and improves the average data rate by $10 \%$.

\section{RELATED WORK}

Several previous works in $\mathrm{mmW}$ communications focused on determining the best channel cluster and communicating through a single beam [2], [3], [12]. Maintaining an active link over a relatively long period of time may not be feasible in this case, due to the dynamic nature of mmW channels and their susceptibility to frequent blockage events [11], [13].

To identify a single channel cluster, three main approaches have been discussed in the literature: Exhaustive search [3], two-stage hierarchical search [2], and context-informationbased (CI-based) search [14]. Exhaustive search is a sequential brute-force approach. It is proposed for $5 \mathrm{G} \mathrm{NR}$, both at the BS and the UE [15]. In the 5G IA process, the BS sequentially transmits synchronization signals along different directions to allow new UEs to detect one of the BS's transmit beams [3]. This exhaustive search comes at the cost of significant discovery time, as each pair of transmit/receive directions must be probed sequentially. On the other hand, the two-stage beam search used in the 802.11ad standard for WiGig devices employs a hierarchical multi-resolution beamforming codebook to reduce the overhead of exhaustive sampling. In the first stage, the access point (AP) sequentially transmits synchronization signals over relatively wide (quasi-omnidirectional) sectors and tries to determine the best coarse direction. In the second stage (beam refinement), the AP refines its search within the selected coarse sector by switching to narrow beams [2]. Although this approach reduces the IA delay, the worst-case search time still scales linearly with the total number of narrow beams. Note that when multiple directions (clusters) are to be found, the performance of hierarchical beam search approaches that of the exhaustive search approach. This is due to the fact that every quasi-omni sector that received signals with a relatively large power in the first stage needs to be scanned with narrow beams in the second stage. Finally, the CI-based search relies on location information. The UE simply selects the closest BS based on location and steers its beam towards the BS [14].

In addition to the above works, others in the literature considered transmissions through multiple antenna lobes. The authors in [16] used a unique approach based on hashing functions to identify the best beam. Although the multi-beam hash functions reduce the search time, they were not used to identify multiple channel clusters. Similar to [16], the authors in [17] also aimed at scanning the environment with multi-lobe beam patterns and establishing communications through multiple lobes. However, they did not optimize their search scheme and simply rotated a pre-determined multilobe pattern to decide the best Rx pattern. As a result, their scheme does not guarantee discovering multiple clusters and combating blockage. Furthermore, the aforementioned works do not consider the tradeoff between simultaneously searching multiple directions and the reduced beamforming gain for a system that utilizes analog beamforming.

In this paper, we develop a novel search scheme called MLBS, which discovers multiple channel clusters in logarithmic time with respect to the number of (narrow) beam directions. We then use these discovered clusters to simultaneously receive copies of the same signal arriving from multiple directions. Phases of the signals coming from different directions are adjusted and the signals are coherently combined. We provide a detailed analysis of the misdetection and false alarm probabilities under MLBS. As the probability that all channel clusters are blocked at the same time is small, our proposed scheme provides an effective mechanism to combat blockage and maintain active communications at acceptable link rates.

\section{System MODEL}

In this paper, we consider the IA process of a mmW system. Electronically steerable phased-array antennas are used at both BS and UEs. In addition, we assume analog beamforming, as it is currently the most energy-efficient beamforming solution available. Without loss of generality, we let the BS be the transmitter (Tx) and UE be the receiver (Rx). In this section, we calculate the array factor ${ }^{1}$ (AF) of the UE antenna array and demonstrate the creation of multi-lobe patterns. Extension to the BS is straightforward.

\section{A. Antenna Arrays}

Consider a UPA that consists of $N$ antenna elements with a horizontal inter-element distance $d_{x}$ and a vertical interelement distance $d_{y}$. Suppose that the incident wave of the received signal arrives at polar angle $\theta$ and azimuth angle $\alpha$ (see Fig. 1), and the antennas are placed on an $N_{x} \times N_{y}$ 2D grid (i.e., $N=N_{x} N_{y}$ ). The phase of the received signal at element

\footnotetext{
${ }^{1}$ The AF is the factor by which the element factor of an individual antenna is multiplied to get the total firing pattern of the entire array.
} 


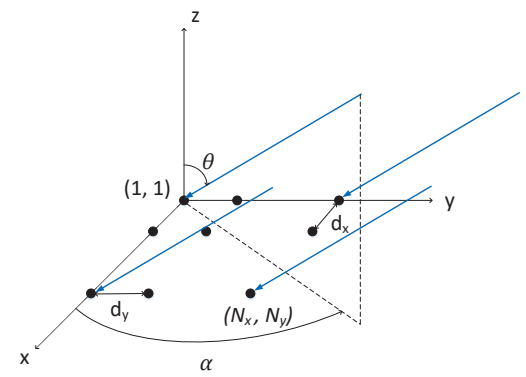

Fig. 1. Visualization of analog Rx beamforming at a UPA (angle of the desired $\mathrm{Rx}$ direction is $(\theta, \alpha)$ ).

$\left(n_{x}, n_{y}\right), n_{x} \in\left\{1, \cdots, N_{x}\right\}$ and $n_{y} \in\left\{1, \cdots, N_{y}\right\}$, leads the phase at element $\left(n_{x}-1, n_{y}-1\right)$ by $2 \pi\left(d_{x} \cos \alpha \sin \theta+\right.$ $\left.d_{y} \sin \alpha \sin \theta\right) / \lambda$, where $\lambda$ is the wavelength of the signal. Let $\kappa \triangleq d_{x} n_{x} \cos \alpha \sin \theta+d_{y} n_{y} \sin \alpha \sin \theta$. The received signal at antenna $\left(n_{x}, n_{y}\right)$ can then be written as:

$$
s_{n_{x}, n_{y}}(\theta, \alpha)=R I_{n_{x}, n_{y}} e^{j \frac{2 \pi}{\lambda} \kappa}
$$

where $I_{n_{x}, n_{y}}$ is the amplitude excitation of the element $\left(n_{x}, n_{y}\right)$ and $R$ is the individual gain of each antenna element. Because in analog beamforming $I_{n_{x}, n_{y}}$ is constant, for simplicity, we let $I_{n_{x}, n_{y}}=1$. Let $w_{n_{x}, n_{y}}$ be a complex phase shifter weight associated with $s_{n_{x}, n_{y}}$. The total received signal is given by:

$$
s(\theta, \alpha)=R \sum_{n_{x}=1}^{N_{x}} \sum_{n_{y}=1}^{N_{y}} w_{n_{x}, n_{y}} e^{j \frac{2 \pi}{\lambda} \kappa}=R F_{\mathrm{UPA}}
$$

where $F_{\mathrm{UPA}}$ is the AF of the UPA. Assuming the same signal magnitude at each antenna, $F_{\mathrm{UPA}}$ can be maximized by selecting $w_{n_{x}, n_{y}}$ in a way to ensure that the received signals are in phase, i.e., by setting $w_{n_{x}, n_{y}}=e^{-j \frac{2 \pi}{\lambda} \kappa}$. This way, the UE beam can be steered along the direction $(\theta, \alpha)$.

To create a pattern with multiple lobes, the antenna array is divided into several sub-arrays. The elements in each subarray are then assigned weights for different steering angles. An example of a two-lobe beam pattern with uniform subarray division is illustrated in Fig. 2, where the steering angles are $\left(\theta_{1}, \alpha_{1}\right)=\left(0^{\circ}, 0^{\circ}\right)$ and $\left(\theta_{2}, \alpha_{2}\right)=\left(30^{\circ}, 0^{\circ}\right)$.

\section{B. Channel Characteristics}

To develop a multi-lobe beamforming design, we must first express the channel between a BS and a UE under UPAs. We use the notation $x \times y$ to denote a matrix of $x$ rows and $y$ columns. Let the total number of antennas at the BS and the UE be $N_{\mathrm{BS}}=N_{\mathrm{BS}, x} \times N_{\mathrm{BS}, y}$ and $N_{\mathrm{UE}}=N_{\mathrm{UE}, x} \times N_{\mathrm{UE}, y}$, respectively. Let $\left(\theta_{p}, \alpha_{p}\right)$ and $\left(\theta_{p}^{\prime}, \alpha_{p}^{\prime}\right)$ be the AoA and angleof-departure (AoD) for cluster $p$ (at the time of reception or transmission, respectively). Also, let $\mathbf{a}_{B}\left(\theta_{p}^{\prime}, \alpha_{p}^{\prime}\right)$ denote the $N_{\text {BS }} \times 1$ array response vector (ARV) of the BS antenna system and $\mathbf{a}_{U}\left(\theta_{p}, \alpha_{p}\right)$ denote the $N_{\mathrm{UE}} \times 1 \mathrm{ARV}$ of the UE antenna system for a given cluster $p$. Then, the $N_{\mathrm{UE}} \times N_{\mathrm{BS}}$ channel

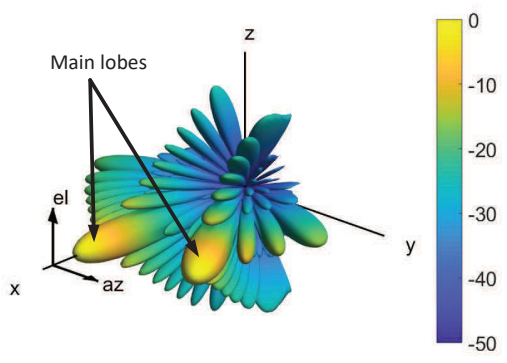

Fig. 2. Normalized 3D directivity pattern of a $16 \times 16$ UPA divided into two sub-arrays, beaming along $\left(\theta_{1}, \alpha_{1}\right)=\left(0^{\circ}, 0^{\circ}\right)$ and $\left(\theta_{2}, \alpha_{2}\right)=\left(30^{\circ}, 0^{\circ}\right)$ (antennas placed on the Y-Z plane).

matrix $\mathbf{H}$ between the $\mathrm{BS}$ and the $\mathrm{UE}$ can be expressed as $\mathbf{H}=$ $\sum_{p=1}^{P} h_{p} \mathbf{a}_{U}\left(\theta_{p}, \alpha_{p}\right) \mathbf{a}_{B}^{*}\left(\theta_{p}^{\prime}, \alpha_{p}^{\prime}\right)$, where $(.)^{*}$ denotes conjugate transpose operation, $P$ is the total number of clusters, and $h_{p}$ is the complex gain of the $p$ th cluster.

The transmit and receive beamforming vectors for the desired directions can be computed offline and stored in the codebooks at the Tx and the Rx. At any time during directional communication, if the BS is using the transmit beamforming vector $\mathbf{f}_{i} \in \mathbb{C}^{N_{\mathrm{BS}} \times 1}$ and UE is using the receive beamforming vector $\mathbf{q}_{j} \in \mathbb{C}^{N_{\mathrm{UE}} \times 1}$ ( $i$ and $j$ are the indices of the beamforming vectors in their codebooks), we can represent the received signal $y_{i j}$ as

$$
y_{i j}=\mathbf{q}_{j}^{*} \mathbf{H} \mathbf{f}_{i} x+\mathbf{q}_{j}^{*} \mathbf{n}
$$

where $x$ is the transmitted signal, and the entries of $\mathbf{n} \in$ $\mathbb{C}^{N_{\mathrm{UE}} \times 1}$ are complex circularly-symmetric white Gaussian noise. Here, $\mathbf{f}_{i}$ and $\mathbf{q}_{j}$ consist of the complex antenna weights $w_{n_{x}, n_{y}}$ (computed as discussed in Section III-A) to construct Tx beam $i$ and Rx beam $j$, respectively. Note that patterns generated by $\mathbf{f}_{i}$ and $\mathbf{q}_{j}$ can have single or multiple main lobes. Let us define the codebook of the Rx beamformer as $\mathcal{Q}$, where $\mathcal{Q}$ consists of $L$ single-lobe beams, $\left(\begin{array}{c}L \\ 2\end{array}\right)$ two-lobe beams, $\cdots$, $\left(\begin{array}{l}L \\ B\end{array}\right) B$-lobe beams, where $B$ is a pre-determined upper-bound on the simultaneous lobes. Here, $L$ is the maximum number of single-lobe beam patterns that can be generated at the Rx, which is a function of the resolution of the phase shifters.

Here, our aim is to find the optimal Rx beamformer that maximizes the average data rate within a time frame, for a given $\mathrm{Tx}$ beamformer. Because $\mathrm{mmW}$ channel clusters experience significant blockages, utilizing several channel clusters simultaneously prevents the Tx and the Rx from losing communication and hence, improve the average data rate. In the next section, we propose the SmartLink protocol that aims at finding the best receive beamformer for a $\mathrm{mmW}$ channel, which is prone to blockage events.

\section{SMARTLink PROTOCOL}

In this section, we explain the main aspects of the SmartLink protocol. Specifically, the protocol consists of three main parts: identifying cluster directions, aligning the phases of the signals 
coming from different paths, and allocating the antennas/power to different clusters optimally, taking blockages into account. In the following subsections, we will cover each item in detail.

\section{A. MLBS Algorithm}

The objective of MLBS algorithm is to identify the directions of the dominant channel clusters at the Rx, for a given Tx direction. Recall that $L$ is the total number of available narrow beam directions. To infer the cluster directions, we propose to take multiple measurements using beam patterns with $B$ lobes. This problem is similar to the game Mastermind, with $L$ colors (directions) and $B$ pegs (simultaneous beams). The outcome of taking a measurement with a pattern at each round will either be a 1 (if the power of the received signal is above a threshold $T$ ) or a 0 (if the power of the received signal is below $T)$. In general, the solution to Mastermind can be found in a relatively small number of rounds using minimax method, i.e., choosing a test pattern that minimizes the maximum number of remaining possibilities at each round. However, finding the optimal solution for the overall problem is NP-hard [18]. As a result, online computation of the optimal sequence of test patterns is not feasible. Thankfully, the optimal strategy can be computed offline considering all possible responses, and stored in a binary decision tree. Then, at each round, the receiver can measure the channel with a pattern, and using the response and the decision tree, it can select the next test pattern optimally.

To build the decision tree, the following method is used. First, an $\left(\begin{array}{l}L \\ P\end{array}\right) \times\left(\begin{array}{l}L \\ B\end{array}\right)$ matrix $\mathbf{D}$ is initialized, where rows correspond to all possible clusters in the environment and columns correspond to all possible $B$-lobe beam patterns. For example, let us consider the row labeled as $(m, n)$ and the column labeled as $(i, j)$. It represents that the $\mathrm{Rx}$ uses a beam pattern with two main lobes at directions $i$ and $j$ to measure the channel that has two clusters along the directions $m$ and $n$. If the Rx pattern captures at least one cluster, the corresponding matrix entry is set to 1 , otherwise it is set to 0 . The proper threshold $(T)$ selection for this binary decision will be discussed in the next section. For the sake of computational and memory efficiency, here we aim at identifying the $B$ strongest clusters. As a result, $\mathbf{D}$ will be a $\left(\begin{array}{l}L \\ B\end{array}\right) \times\left(\begin{array}{l}L \\ B\end{array}\right)$ matrix. In case that the channel exhibits fewer clusters, algorithm will still return $B$ output directions, but no antenna/power will be allocated for the weakest cluster.

Without loss of generality, the root of the decision tree is selected as pattern $(1,2, \cdots, B)$, i.e., the pattern with $B$ main lobes in the directions $1, \cdots, B$. Once a measurement result for the initial pattern is obtained, the next pattern is selected in a way to divide the remaining cluster directions as evenly as possible, to minimize the maximum remaining possibilities. Specifically, next test pattern should have even (or close to even) number of $1 \mathrm{~s}$ and $0 \mathrm{~s}$ for the remaining possible cluster directions. This way, the remaining candidate directions are halved at each round, until the unique cluster directions are identified. As a result, although the complexity of constructing the decision tree from matrix $\mathbf{D}$ is exponential, it could be done once and offline. Once the matrix is constructed,

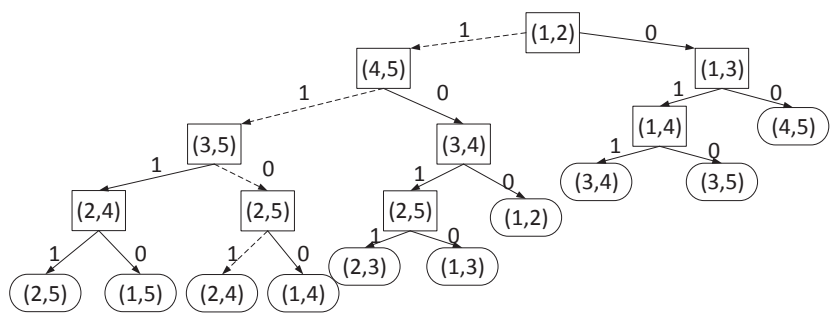

Fig. 3. Decision tree for matrix D. Path traversed in the example is shown with dashed lines.

identifying the cluster directions take logarithmic time, as the remaining possibilities are reduced approximately by half at each iteration. On the other hand, exhaustive 5G beam search scales linearly with $L$, regardless of how many clusters the BS and the UE aim at finding, as they probe each beam direction sequentially. Finally, 802.11ad search also scales linearly with $L$. Even though its search time may be relatively low when the aim is to find a single cluster, the search time may even be larger than exhaustive search when the algorithm aims at finding multiple clusters. It is simply due to the fact that each quasi-omni beam with an Rx power that is larger than $T$ needs to be searched with narrow beams, and in the worst case, all quasi-omni beams return Rx powers larger than $T$.

Example: Here, we illustrate how the algorithm runs with an example, where $L=5$ and $B=2$. For this case, the matrix $\mathbf{D}$ can be constructed as in Table I. Notice that when at least one number in the row and column tuples overlap, i.e., a cluster is captured with a pattern, the corresponding entry of the matrix is set to 1 .

TABLE I

DECISION MATRIX FOR $L=5$ AND $B=2$.

\begin{tabular}{c|cccccccccc} 
& $(1,2)$ & $(1,3)$ & $(1,4)$ & $(1,5)$ & $(2,3)$ & $(2,4)$ & $(2,5)$ & $(3,4)$ & $(3,5)$ & $(4,5)$ \\
\hline$(1,2)$ & 1 & 1 & 1 & 1 & 1 & 1 & 1 & 0 & 0 & 0 \\
$(1,3)$ & 1 & 1 & 1 & 1 & 1 & 0 & 0 & 1 & 1 & 0 \\
$(1,4)$ & 1 & 1 & 1 & 1 & 0 & 1 & 0 & 1 & 0 & 1 \\
$(1,5)$ & 1 & 1 & 1 & 1 & 0 & 0 & 1 & 0 & 1 & 1 \\
$(2,3)$ & 1 & 1 & 0 & 0 & 1 & 1 & 1 & 1 & 1 & 0 \\
$(2,4)$ & 1 & 0 & 1 & 0 & 1 & 1 & 1 & 1 & 0 & 1 \\
$(2,5)$ & 1 & 0 & 0 & 1 & 1 & 1 & 1 & 0 & 1 & 1 \\
$(3,4)$ & 0 & 1 & 1 & 0 & 1 & 1 & 0 & 1 & 1 & 1 \\
$(3,5)$ & 0 & 1 & 0 & 1 & 1 & 0 & 1 & 1 & 1 & 1 \\
$(4,5)$ & 0 & 0 & 1 & 1 & 0 & 1 & 1 & 1 & 1 & 1
\end{tabular}

Following the previously explained procedure and using $\mathbf{D}$, the decision tree of this matrix can be constructed as in Fig. 3 . Note that the decision tree is not unique. Fig. 3 visualizes one of the optimal decision trees that has the minimum depth. In the decision tree, the rectangles correspond to actions (i.e., patterns to be measured), the ellipses correspond to results (i.e., inferred directions), and left and right branches correspond to a measurement outcome of 1 or 0 , respectively.

Let the strongest clusters that the algorithm aims at discovering be in the directions 2 and 4 (i.e., the row labeled as $(2,4))$. The algorithm starts with the initial pattern $(1,2)$, as explained before. Since pattern $(1,2)$ will capture cluster 2 , the outcome of this measurement will be 1 . As a result, 
last three rows of $\mathbf{D}$ will be eliminated, meaning that the two clusters that we are trying to discover cannot be $(3,4),(3,5)$ or $(4,5)$. Next, the algorithm proceeds with another pattern that contains an (almost) even number of $1 \mathrm{~s}$ and $0 \mathrm{~s}$ in the remaining rows. For that purpose, pattern $(4,5)$ can be chosen, as it contains three $0 \mathrm{~s}$ and four $1 \mathrm{~s}$ in the top seven rows. Since this pattern also captures a cluster (in direction 4), the result will again be 1 . After this step, only four rows remain: $(1,4)$, $(1,5),(2,4)$ and $(2,5)$. Next, the pattern $(3,5)$ can be chosen, as it divides the remaining rows evenly. The outcome of this measurement will be 0 , leaving two remaining rows $(1,4)$ and $(2,4)$. The algorithm then proceeds with the final measurement using pattern $(2,5)$, and using its outcome, cluster directions can be uniquely identified as $(2,4)$, in just four steps. $5 \mathrm{G}$ beam search, on the other hand, requires measuring all five directions to find the clusters. Finally, 802.11ad search will initially take two measurements using quasi-omni beams (say, $(1,2,3)$ and $(4,5)$ ), and then will do beam refinement in both of these quasi-omni beams. As a result, with seven measurements total, it will take even longer time compared to exhaustive search. Clearly, the reduction in discovery time that MLBS provides is more prominent when $L \gg 5$, which is typical for a $\mathrm{mmW}$ system.

MLBS algorithm can be easily integrated into $5 \mathrm{G}$ devices. In $5 \mathrm{G}$, the BS periodically broadcasts primary and secondary synchronization signals [19] towards pre-defined number of beam directions in a sequential manner. UEs utilize these signals to establish a new connection with the BS and/or to perform beam searching. However, UEs have limited time to detect a synchronization signal transmitted towards a specific direction, before the BS switches its beam direction. Implementation of the beam searching method at the UE side is not standardized [19]. Therefore, MLBS can be employed in 5G UEs without requiring any modification to the standards, and would allow the UEs to discover the best Rx beam directions for a given BS beam.

\section{B. Threshold Selection}

As explained in the previous section, MLBS algorithm utilizes received signal power, $P_{\mathrm{Rx}}$, to determine whether any cluster is captured or not by a given antenna pattern. In particular, if $P_{\mathrm{Rx}} \geq T,\left(P_{\mathrm{Rx}}<T\right)$ the binary outcome of the measurement is set to $1(0)$. However, due to the multipath effects of the channel and the side-lobes of Tx and Rx beams, undesired decisions can be given depending on the selection of $T$. For example, if $T$ is small, 1 may be observed even when Tx and Rx beams are not perfectly aligned. On the other hand, when an antenna pattern with $B$ main lobes captures more than one signal coming from different paths, the signals may add up destructively due to their random arrival phases and the measurement outcome may be 0 (even though it should be 1). Therefore, $T$ must be selected in a way to account for these false positives (false alarm) and false negatives (misdetection). Examples of false positives and false negatives are visualized in Fig. 4.

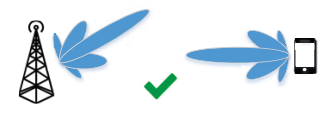

(a)

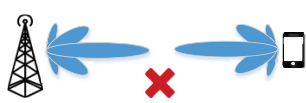

(b)
Fig. 4. Scenarios with wrong decisions: (a) false positive: we decide on PASS even though Tx and Rx beams are not aligned, (b) false negative: we decide on FAIL even though Tx and Rx beams are aligned.

Here, we apply a likelihood-ratio test based on $P_{\mathrm{Rx}}$ to decide whether the current measurement outcome should be set to 1 or 0 . In particular, our hypothesis testing includes the following two events:

- $H_{1}$ : at least one cluster is captured by the main lobes of a given pattern,

- $H_{0}$ : clusters are captured only by the side lobes of a given pattern, or no cluster is captured at all.

To properly choose a threshold value $T$, we employ NeymanPearson hypothesis testing and evaluate the misdetection probability, $\operatorname{Pr}\{\mathrm{MD}\}$, and the false alarm probability, $\operatorname{Pr}\{\mathrm{FA}\}$. Note that $\operatorname{Pr}\{\mathrm{MD}\}=\operatorname{Pr}\left\{P_{\mathrm{Rx}}<T \mid H_{1}\right\}$ and $\operatorname{Pr}\{\mathrm{FA}\}=$ $\operatorname{Pr}\left\{P_{\mathrm{Rx}} \geq T \mid H_{0}\right\}$. Neyman-Pearson test can be expressed by the following optimization problem:

$$
\begin{array}{cl}
\underset{T}{\operatorname{minimize}} & \operatorname{Pr}\{\mathrm{MD}\} \\
\text { s.t. } & \operatorname{Pr}\{\mathrm{FA}\} \leq \gamma_{\mathrm{FA}}
\end{array}
$$

where $\gamma_{\mathrm{FA}}$ is a given maximum tolerable false alarm probability. From the definition, it is easy to see that $\operatorname{Pr}\{M D\}$ $(\operatorname{Pr}\{\mathrm{FA}\})$ is a monotonically increasing (decreasing) function with respect to $T$. Therefore, to minimize $\operatorname{Pr}\{\mathrm{MD}\}$, the smallest $T$ should be chosen while satisfying the constraint in (4). Hence, the optimal $T$ value is obtained when the inequality in (4) holds with equality.

Let $T^{\prime}$ indicate the optimal threshold value, i.e., $\operatorname{Pr}\{\mathrm{FA}\}=$ $\operatorname{Pr}\left\{P_{\mathrm{Rx}} \geq T^{\prime} \mid H_{0}\right\}=\gamma_{\mathrm{FA}}$. To find $T^{\prime}$, we need to evaluate $P_{\mathrm{Rx}}$. For analytical tractability, the actual antenna patterns are approximated by a sectored antenna model as commonly used in the literature [20]-[22]. Specifically, let $G(\phi)$ be the antenna gain of Rx-antenna array where $\phi \in[0,2 \pi]$ is the angle off the broadside direction. Then,

$$
G(\phi)= \begin{cases}G_{\max } & \text { if }\left|\phi-\phi_{b}\right| \leq \omega / 2, b \in\{1, \cdots, B\} \\ G_{\min } & \text { otherwise }\end{cases}
$$

where $\omega \in[0,2 \pi]$ is the beamwidth of a main lobe, $\phi_{b} \in$ $[0,2 \pi], \forall b \in\{1, \cdots, B\}$, is the direction of $b$ th main lobe, and $G_{\max }$ and $G_{\min }$ are the antenna gains at the main and side lobes, respectively.

Let $r_{p} e^{j \varphi_{p}}$ be the received signal from the $p$ th cluster, where $r_{p}$ and $\varphi_{p}$ represent its magnitude and phase, respectively. $P_{\mathrm{Rx}}$ is then given by:

$$
P_{\mathrm{Rx}}=\left(\sum_{p \in \mathcal{P}} r_{p} e^{j \varphi_{p}}\right)\left(\sum_{p \in \mathcal{P}} r_{p} e^{j \varphi_{p}}\right)^{*}+P_{N}
$$


where $P_{N}=n n^{*}$ and $n \sim \mathcal{C N}\left(0, \sigma_{N}^{2}\right)$ due to the additive white Gaussian noise. In the analysis, we rely on the 3GPP channel model as described in [23]. Based on this model, the phase of a signal received from a particular cluster is a uniform random variable from 0 to $2 \pi$, i.e., $U(0,2 \pi)$. Let us evaluate the received signal power from the $p$ th cluster, i.e., $r_{p}^{2}$. Let $P_{p}$, $P_{\text {Tx }}$, and $P_{L}$ denote the $p$ th cluster power, the transmit power of BS (including the Tx antenna array gain), and the path loss of the channel between BS and UE, respectively. Based on the sectored antenna model, $H_{0}$ is the event in which all the dominant channel clusters between the BS and the UE are captured by the side lobe. Therefore, given that $H_{0}$ occurs, $r_{p}^{2}$ is given by:

$$
r_{p}^{2}=G_{\min } P_{p} P_{\mathrm{Tx}} P_{L} .
$$

In $\mathrm{dB}$ scale, the path-loss can be modeled as $-10 \log _{10}\left(P_{L}\right)=$ $\alpha+10 \beta \log _{10}(d)+\xi$ where $d$ is the distance between the $\mathrm{BS}$ and the UE, $\alpha$ and $\beta$ are frequency and environment dependent constants, and $\xi \sim \mathcal{N}\left(0, \sigma_{S F}^{2}\right)$ is the shadow fading. According to [23], the cluster powers are calculated as follows. Let $X_{p} \sim U(0,1), \forall p \in \mathcal{P}$. Then, $P_{p}^{\prime}$ is defined as $P_{p}^{\prime} \triangleq X_{p}^{\tau_{p}-1} 10^{-0.1 Z_{p}}$ where $Z_{p} \sim \mathcal{N}\left(0, \zeta^{2}\right), \forall p \in \mathcal{P} . \tau_{p}$ and $\zeta$ are known constants that depend on the underlying scenario. Finally, the cluster power $P_{p}$ is given by:

$$
P_{p}=\frac{P_{p}^{\prime}}{\sum_{n \in \mathcal{P}} P_{n}^{\prime}} .
$$

It is not possible to obtain a tractable analysis with the constraint in (4) due to the unknown CDF of the false alarm probability. Hence, we exploit Markov's inequality, which states that $\operatorname{Pr}\{X \geq a\} \leq \mathbb{E}[X] / a$ for a nonnegative random variable $X$ and $a>0$. In particular, $\operatorname{Pr}\left\{P_{\mathrm{Rx}} \geq T^{\prime} \mid H_{0}\right\} \leq$ $\mathbb{E}\left[P_{\mathrm{Rx}} \mid H_{0}\right] / T^{\prime}$. Here, the expectation is over $\varphi_{p}, n, d, \xi$, $X_{p}$, and $Z_{p}, \forall p \in \mathcal{P}$. Note that these random variables are independent. Let us define $\varphi_{k l} \triangleq \varphi_{k}-\varphi_{l}$ where $k, l \in \mathcal{P}$. Thus,

$$
\begin{aligned}
& \mathbb{E}\left[P_{\mathrm{Rx}} \mid H_{0}\right]=\mathbb{E}\left[\left(\sum_{p \in \mathcal{P}} r_{p} e^{j \varphi_{p}}\right)\left(\sum_{p \in \mathcal{P}} r_{p} e^{j \varphi_{p}}\right)^{*}+P_{N}\right] \\
& =\sum_{p=1}^{P} \mathbb{E}\left[r_{p}^{2}\right]+2 \sum_{k=1}^{P} \sum_{l=1}^{k-1} \mathbb{E}\left[r_{k} r_{l}\right] \mathbb{E}\left[\cos \left(\varphi_{k l}\right)\right]+\sigma_{N}^{2} \\
& =P G_{\min } P_{\mathrm{Tx}} 10^{-0.1 \alpha} \mathbb{E}\left[P_{p}\right] \mathbb{E}\left[d^{-\beta}\right] \mathbb{E}\left[10^{-0.1 \xi}\right] \\
& \quad+\sigma_{N}^{2} \\
& =G_{\min } P_{\mathrm{Tx}} 10^{-0.1 \alpha} \mathbb{E}\left[d^{-\beta}\right] \mathbb{E}\left[10^{-0.1 \xi}\right]+\sigma_{N}^{2} \\
& =G_{\min } P_{\mathrm{Tx}} 10^{-0.1 \alpha} \mathbb{E}\left[d^{-\beta}\right] e^{\frac{\left(0.1 \sigma_{S F} \ln (10)\right)^{2}}{2}}+\sigma_{N}^{2} .
\end{aligned}
$$

Here, (9) follows from the independence of signal phases and their magnitudes. (10) follows from $\mathbb{E}\left[\cos \left(\varphi_{k l}\right)\right]$ being 0 as $\varphi_{k} \sim U[0,2 \pi]$ and $\varphi_{l} \sim U[0,2 \pi] \forall k, l \in \mathcal{P}$. Furthermore, we replace $r_{p}^{2}$ by the expression in (7) and use the independence of underlying random variables. Also, note that all clusters have the same $P_{p}, d$, and $\xi$ distributions, so $\sum_{p=1}^{P} \mathbb{E}\left[r_{p}^{2}\right]=$
$P \mathbb{E}\left[r_{p}^{2}\right]$. (11) follows from $\mathbb{E}\left[P_{p}\right]=1 / P$ due to (8). Finally, (12) follows from the definition of the log-normal distribution.

IA is performed when a new UE enters the range of the BS, or a UE attempts to change its status from idle to connected. Therefore, instead of relying on specific distributions for UE locations, the maximum possible distance between the BS and a UE, $D_{\max }$, can be considered in the equations above. In that case, $\mathbb{E}\left[d^{-\beta}\right]$ can be replaced by $D_{\max }^{-\beta}$ in (12). Alternatively, it can be assumed that UEs are randomly and uniformly located on a ring around the BS. Hence, the CDF of the distance between the $\mathrm{BS}$ and a UE is given by $\operatorname{Pr}[D<d]=$ $\frac{d^{2}-D_{\min }^{2}}{D_{\max }^{2}-D_{\min }^{2}}$ where $D_{\min }$ is the minimum distance between the $\mathrm{BS}$ and the UE, and $d \in\left[D_{\min }, D_{\max }\right]$. As a result, $\mathbb{E}\left[d^{-\beta}\right]$ can be replaced by $2\left(D_{\max }^{(2-\beta)}-D_{\min }^{(2-\beta)}\right) /\left((2-\beta)\left(D_{\max }^{2}-D_{\min }^{2}\right)\right)$ in (12).

Combining all the results obtained in this section, $T$ should be set to $\mathbb{E}\left[P_{\mathrm{Rx}} \mid H_{0}\right] / \gamma_{\mathrm{FA}}$ to achieve the minimum misdetection probability for a given $\gamma_{\mathrm{FA}}$. Note that $T$ does not depend on the number of clusters, $P$.

\section{Phase Alignment}

After the cluster directions are discovered, we then need to determine the phases and magnitudes of the signals arriving the Rx through each cluster, in order to add the multipath components coherently. This process is similar to how Rake receivers work [24]. A rake receiver is designed to cope with the effects of multipath fading and it uses several sub-receivers called fingers to achieve it. In particular, each finger is assigned to a different multipath component, and independently performs decoding. Then, the contribution of all fingers are coherently combined and processed altogether. However, this method requires multiple fingers and results in a significantly more complex system compared to a single-RF-chain receiver. Here, as the cluster directions are already determined previously, instead of employing a separate finger per multipath component, each cluster is probed with the optimal beam for that direction, in a different time slot. The received signals are then decoded, and the phases and magnitudes of the signals coming from each cluster are computed.

To coherently add the signals, the phase alignment problem with $B$ beam directions can be more formally written as:

$$
\begin{array}{cl}
\underset{\mathbf{c}}{\operatorname{maximize}} & \left|c_{1} \mathbf{q}_{\phi_{1}}^{*} \mathbf{g}+\cdots+c_{B} \mathbf{q}_{\phi_{B}}^{*} \mathbf{g}\right| \\
\text { s.t. } & \left|c_{i}\right|=1, \forall i \in\{1, \cdots, B\}
\end{array}
$$

where $\phi_{b}$ denotes the direction of the beam $b, \forall b \in$ $\{1, \cdots, B\}$, and $\mathbf{q}_{\phi_{b}}$ represents the beamforming vector that forms a beam towards $\phi_{b}$. Here, the objective function is the magnitude of the superimposed signals received from all $B$ beams, optimization variable is the vector of unit-modulus complex weights, and the constraint is a result of analog beamforming (having only phase shifters and no amplifiers). As stated above, after separately probing $B$ clusters, we acquire their phases and magnitudes. Therefore, by setting the first cluster as reference, i.e., $c_{1}=1$, we can easily compute $c_{i} \forall i \in\{2, \cdots, B\}$. In particular, $c_{2}=e^{j\left(\varphi_{1}-\varphi_{2}\right)}$ so that the 
phases of two signals coming from the first and second beams are aligned. The magnitudes of the signals will be utilized in the next subsection.

Note that the phase alignment is only required after the signal directions are found. Thus, (13) needs to be solved only once, after the clusters are determined. The alignment of phases is not possible with 802.11ad search as all antennas are used to create a single main-lobe, which can capture more than one signal adding destructively [25].

\section{Optimal Antenna Allocation}

For analytical tractability, we consider a uniform linear array (ULA) in this section. A ULA steers a beam in a certain direction with a power that is directly proportional to its number of antennas under half-wavelength spacing (which is the recommended value by the 3GPP standards [23]). Let us now consider the case where the ULA receives the same information signal from $B$ clusters/directions, where $N_{b}$ denotes the number of antennas at the subarray that is devoted to the $b$ th cluster. Existing approaches find the most dominant cluster and allocate all antennas to its direction. Hence, the received signal power $P_{\mathrm{Rx}}=N_{\mathrm{UE}} P_{b} P_{\mathrm{Tx}} P_{L}+P_{N}$, as $B=1$. However, we propose to dynamically divide the antenna array into subarrays, such that each subarray forms a beam towards the direction of a different cluster. Assuming that the phase alignment is achieved as explained in Section IV-C, $P_{\mathrm{Rx}}=\left(\sum_{b \in \mathcal{B}} N_{b} K_{b}\right)^{2}+P_{N}$ where $K_{b} \triangleq \sqrt{P_{b} P_{\mathrm{Tx}} P_{L} / N_{\mathrm{UE}}}$ $\forall b \in \mathcal{B} \triangleq\{1, \cdots, B\}$. By coherently combining signals from multiple clusters, we aim at achieving higher average transmission rate between the BS and the UE under the dynamic blockage scenarios. This way, even when one (or several) of the clusters is blocked, the Tx-Rx link will not be lost.

Dynamic blockage in wireless systems is commonly modeled by a Poisson process [10], [11]. Thus, the arrival time of a blocker is exponentially distributed. Here, we assume that the blockers are impenetrable, and the blockage process of each link/cluster is independent and identically distributed. Let $\rho$ denote the blockage frequency, i.e., the rate parameter of the exponential distribution. The formulation of the associated optimization problem depends on $B$, so for simplicity, we provide the formulation for the case of $B=2$. Let $t_{1}$ denote the time until the one of clusters is blocked since the beginning of the transmission. Similarly, let $t_{2}$ denote the time until the blockage of the second cluster, after the first one is blocked. Hence, the problem of maximizing the total transmitted data until all the clusters are blocked is formulated by:

$$
\begin{aligned}
\underset{N_{b}, \forall b \in \mathcal{B}}{\operatorname{maximize}} & \mathbb{E}\left[t_{1} \log _{2}\left(U_{\mathcal{B}}\right)+t_{2} \log _{2}\left(U_{l}\right)\right] \\
\text { s.t. } & \sum_{b \in \mathcal{B}} N_{b}=N_{\mathrm{UE}}, \quad N_{b} \in\{0\} \cup \mathbb{Z}^{+} \quad \forall b \in \mathcal{B}
\end{aligned}
$$

Here, $U_{\mathcal{B}} \triangleq 1+\left(\sum_{b \in \mathcal{B}} N_{b} K_{b}\right)^{2} / \sigma_{N}^{2}$ and $U_{l} \triangleq 1+N_{l}^{2} K_{l}^{2} / \sigma_{N}^{2}$ where $l$ th cluster represents the last blocked cluster. The expectation in (14) is taken with respect to $t_{1}$ and $t_{2}$. Due to the merging and memoryless properties of Poisson process, $\mathbb{E}\left[t_{1}\right]=(2 \rho)^{-1}$ and $\mathbb{E}\left[t_{2}\right]=\rho^{-1}$. Hence, expectation in the objective function can be eliminated and we obtain $(2 \rho)^{-1} \log _{2}\left(1+\left(\sum_{b \in \mathcal{B}} N_{b} K_{b}\right)^{2} / \sigma_{N}^{2}\right)+(2 \rho)^{-1} \log _{2}(1+$ $\left.\left(N_{1} K_{1}\right)^{2} / \sigma_{N}^{2}\right)+(2 \rho)^{-1} \log _{2}\left(1+\left(N_{2} K_{2}\right)^{2} / \sigma_{N}^{2}\right)$. Note that the probability of the first cluster being blocked before the other one is 0.5 (and vice versa). As a result, the final optimization is a nonlinear integer programming problem, which is NPhard. However, it can be converted to a convex optimization problem, if the integer restrictions of (14) are relaxed such that $N_{b}$ 's are allowed to take any nonnegative real number, i.e., $N_{b} \geq 0, \forall b \in \mathcal{B}$. Particularly, the objective and constraint functions of this relaxed problem are concave and convex with respect to $N_{b} \forall b \in \mathcal{B}$, respectively. Hence, this problem can be numerically and efficiently solved using existing methods, such as the gradient descend. Afterwards, that solution can be used to determine $N_{b}$ 's of the original problem. Even though it does not guarantee the optimal solution, $N_{b}$ 's can be set to $\left\lceil N_{b}^{*}\right\rceil$ or $\left\lfloor N_{b}^{*}\right\rfloor \forall b \in \mathcal{B}$, such that $\sum_{b \in \mathcal{B}} N_{b}=N_{\mathrm{UE}}(\lceil$.$\rceil and$ $\lfloor$. $\rfloor$ are ceiling and floor functions, respectively). This way, the integer restrictions can be satisfied. Note that the solution of the relaxed problem provides an upper-bound for the original problem. We can compare the performance gap between this upper-bound and the one obtained by using ceiling and floor functions. If the gap is relatively small, the obtained solution may be good enough. Alternatively, we can apply branch and bound algorithm, which is one of the most common methods to solve integer programming problems. This method exploits the solution of the relaxed problem to add certain bounding constraints to the original one. Although these extra constraints lead to the optimal solution, which is purely integer, the complexity of the algorithm is high. Thankfully, given that $B$ is typically a small number, the size of our problem in (14) is not large, meaning that branch and bound method can be efficiently applied here. Note that the problem formulation in the case of different $B$ values is straightforward and omitted here for brevity.

\section{Performance Evaluation}

In this section, we evaluate the performance of Smartlink through extensive trace-driven emulations. We compare Smartlink with 802.11ad-like and 5G beam search approaches, in which the search time scales linearly with $L$.

\section{A. Experimental Setup}

We first conduct extensive experiments to obtain the $\mathrm{mmW}$ channel characteristics to be used in our simulations. $4 \times 4$ UPAs are used in our experiments with $d_{x}=0.5 \lambda$ and $d_{y}=0.6 \lambda$. The antenna gain at the broadside of the array is $12 \mathrm{~dB}$. For the sake of measuring the received power, a continuous wave with $5 \mathrm{dBm}$ amplitude is transmitted over the $29 \mathrm{GHz}$ band, which is a candidate band for $5 \mathrm{G}$ communications. Keysight E8257D-ATO-8384 PSG signal generator is used to generate the waveform. At the Rx side, the array is connected to Keysight PXA-550-MY55002004 vector signal analyzer (VSA). To steer the transmit/receive beams to desired 


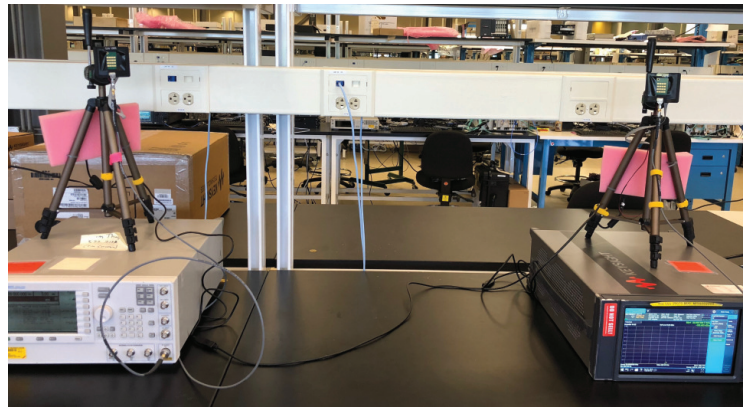

Fig. 5. Test setup using $4 \times 4$ UPAs both at the Tx and the Rx side, signal generator and vector signal analyzer.

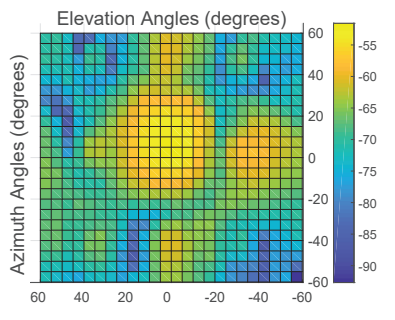

(a)

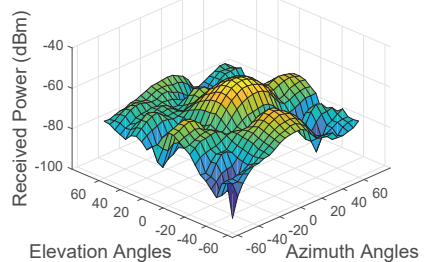

(b)
Fig. 6. Rx powers for different beam directions at $29 \mathrm{GHz}$ operating frequency and under first scenario (Tx-Rx seperation of $3 \mathrm{~m}$ ). (a) Flattened, (b) 3D representation.

directions, antenna arrays are connected to microcontrollers, which are interfaced with the PC through serial port. The whole setup with the Tx, Rx, PSG, and the VSA can be seen in Fig. 5.

We test several LOS and NLOS scenarios with a Tx-Rx separation of $3 \mathrm{~m}$, where the NLOS path is created by a 1.2 $\mathrm{m} \times 1.2 \mathrm{~m}$ metal reflector. The effective beam scanning range of the UPAs in our experiments are $\pm 60^{\circ}$ from broadside, in both azimuth and elevation (effective FOV $=\sim 120^{\circ}$ in each direction). Beyond that, the antenna gain drops significantly as a result of the non-ideal behavior of the antenna elements. To experimentally obtain the AoA profile, we exhaustively scanned the 3D space within the effective beam scanning range of the antenna arrays under different scenarios. Using the above setup, we collected RSS measurements from the 3D space. A sample plot is shown in Fig. 6, where the beam is swept in $5^{\circ}$ steps.

In Fig. 7(a), we evaluate the effect of the threshold $T$ that is used in MLBS algorithm when $B=3$. We take the average values obtained from all LOS and NLOS scenarios. As expected, when $T$ increases, $\operatorname{Pr}\{\mathrm{MD}\}$ also increases and $\operatorname{Pr}\{\mathrm{FA}\}$ decreases. Thus, for this experimental environment, when $T$ is approximately equal to $-68 \mathrm{dBm}$, both $\operatorname{Pr}\{\mathrm{MD}\}$ and $\operatorname{Pr}\{\mathrm{FA}\}$ stay below 0.2 . We then compare the throughput performances of a benchmark scheme (BM) that allocates all antennas to form a beam towards the strongest cluster and SmartLink, under certain blockage probabilities of the underlying links. In Fig. 7(b), it is shown that SmartLink outperforms $\mathrm{BM}$ up to $10 \%$. The results also indicate that

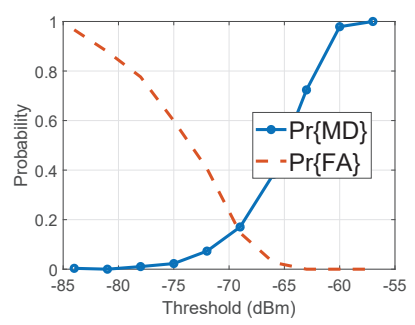

(a)

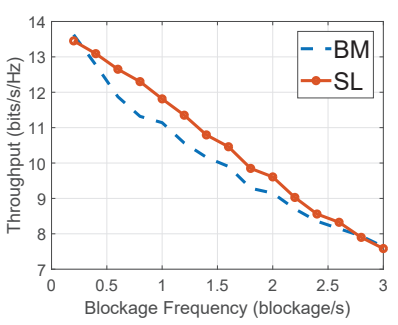

(b)
Fig. 7. Trace-driven simulation results: (a) comparison of misdetection and false alarm probabilities for a given threshold, (b) throughput vs. blockage frequency (comparison of a benchmark scheme and SmartLink).

when the blockage frequency is low, BM performs as good as SmartLink. In that case, the $\mathrm{Tx}$ and $\mathrm{Rx}$ beams are realigned until or right after the blockage happens. Note that when we allocate the antennas to form multiple beams, the instantaneous transmission rate decreases. However, under the dynamic blockage, SmartLink allows an uninterrupted transmission due to the utilization of multiple beams. This leads to a higher average transmission rate when the blockage frequency is between 0.2 and 2.8 blockage per second (bl/s). When the blockage frequency is higher than $2.8 \mathrm{bl} / \mathrm{s}$, the performances of both schemes become the same. In that case, all clusters used in SmartLink are prone to be blocked in a short amount of time due to the high blockage frequency. Hence, the advantage of SmartLink over BM disappear.

\section{B. Simulation Results}

In simulation results, we use 3GPP channel models [23] where $P_{\mathrm{Tx}}=46 \mathrm{dBm}$, the operating frequency is $28 \mathrm{GHz}$, the bandwidth is $57.6 \mathrm{MHz}$, and the cell radius is $200 \mathrm{~m}$. A ULA of half-wavelength antenna spacing is implemented with various numbers of antennas.

A numerical comparison of MLBS with 5G and 802.11adlike beam searching schemes are shown in Fig. 8. 5G beam search corresponds to the traditional exhaustive beam scan and 802.11ad-like search represents the hierarchical beam search that aims at identifying multiple channel clusters. Clearly, discovery times of all schemes increase with increasing $L$. In Fig. 8 (a), MLBS decreases the discovery time by $65 \%$ compared to 5G beam scan and by $30 \%$ compared to 802.11 ad-like scheme. Note that compared to Fig. 8(a), curves in Fig. 8(b) are closer to each other. Naturally, as the clusters to be discovered approaches $L$, all schemes converge to the exhaustive beam scan. However, due to fewer reflections in $\mathrm{mmW}$ spectrum, only a few channel clusters are present in $\mathrm{mmW}$ channels.

In Fig. 9(a), the comparison of $\operatorname{Pr}\{\mathrm{MD}\}$ and $\operatorname{Pr}\{\mathrm{FA}\}$ for various number of antennas is provided, when $B=3$. The results are obtained for various $\gamma_{\mathrm{FA}}$ values that is used to determine the threshold $T$ as explained in Section IV-B. It shows that when the antenna gain per cluster increases, $\operatorname{Pr}\{\mathrm{MD}\}$ decreases. Also note that $\operatorname{Pr}\{\mathrm{FA}\}$ does not change with $N_{\mathrm{UE}}$. Fig. 9(b) demonstrates the effect of blockage rate 


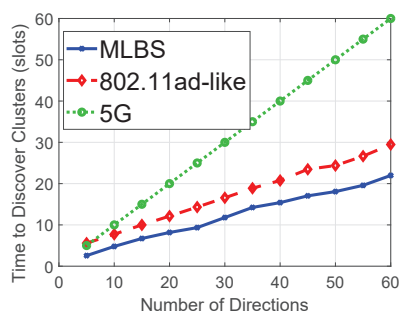

(a)

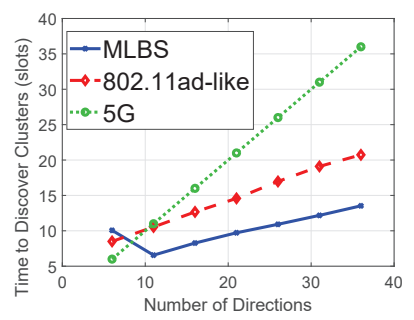

(b)
Fig. 8. Comparison of cluster discovery times between MLBS, 5G and 802.11ad-like beam search approaches. (a) Discovering 2 clusters, (b) Discovering 3 clusters.

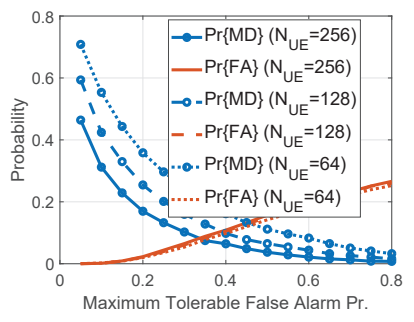

(a)

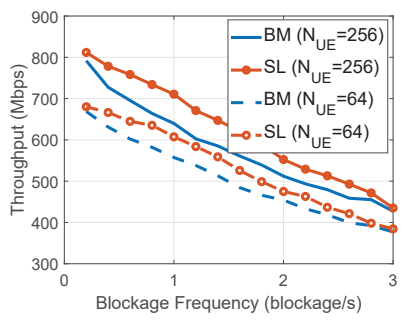

(b)
Fig. 9. Numerical results: (a) comparison of misdetection and false alarm probabilities for various number of lobes and antennas, (b) throughput vs. blockage frequency (comparison of a benchmark scheme and SmartLink).

on the throughput. The results here verify the ones in Fig. 7(b), obtained via experiments.

\section{CONCLUSiOnS}

In this paper, we proposed an efficient communication protocol for $\mathrm{mmW}$ systems called SmartLink. SmartLink utilizes multiple channel clusters in the $\mathrm{mmW}$ channel between the BS and the UE to combat blockage. It uses a novel search scheme called MLBS, which discovers multiple clusters in logarithmic time with respect to the number of beam directions. Discovered clusters are then simultaneously used for transmission/reception, and the signals coming from different directions are coherently combined. As the probability of all channel clusters being blocked at the same time is low, SmartLink provides an effective mechanism to maintain communications and improve the average data rate. Our future work will focus on multi-lobe beam searching methods under hybrid beamforming architectures.

\section{REFERENCES}

[1] T. S. Rappaport, S. Sun, R. Mayzus, H. Zhao, Y. Azar, K. Wang, G. N. Wong, J. K. Schulz, M. Samimi, and F. Gutierrez, "Millimeter wave mobile communications for 5G cellular: It will work!" IEEE Access, vol. 1, pp. 335-349, 2013.

[2] IEEE Computer Society, "IEEE Standard-part 11: Wireless LAN medium access control (MAC) and physical layer (PHY) specifications amendment 3: Enhancements for very high throughput in the $60 \mathrm{GHz}$ band (adoption of IEEE std 802.11ad-2012)," 2014. [Online]. Available: https://standards.ieee.org/findstds/standard/802.11ad-2012.html.

[3] 3GPP TR 38.802 v14.2.0, "Study on new radio access technologyphysical layer aspects (release 14)," Sep. 2017. [Online]. Available: http://www.3gpp.org/ftp//Specs/archive/38series/38.802/38802e20.zip.
[4] J. G. Andrews, S. Buzzi, W. Choi, S. V. Hanly, A. Lozano, A. C. Soong, and J. C. Zhang, "What will 5G be?" IEEE J. Sel. Areas Commun., vol. 32, no. 6, pp. 1065-1082, 2014.

[5] M. R. Akdeniz, Y. Liu, M. K. Samimi, S. Sun, S. Rangan, T. S. Rappaport, and E. Erkip, "Millimeter wave channel modeling and cellular capacity evaluation," IEEE J. Sel. Areas Commun., vol. 32, no. 6, pp. 1164-1179, 2014.

[6] O. El Ayach, S. Rajagopal, S. Abu-Surra, Z. Pi, and R. W. Heath, "Spatially sparse precoding in millimeter wave MIMO systems," IEEE Trans. Wireless Commun., vol. 13, no. 3, pp. 1499-1513, 2014.

[7] C. N. Barati, S. A. Hosseini, S. Rangan, P. Liu, T. Korakis, S. S. Panwar, and T. S. Rappaport, "Directional cell discovery in millimeter wave cellular networks," IEEE Trans. Wireless Commun., vol. 14, no. 12, pp. 6664-6678, 2015.

[8] I. Aykin and M. Krunz, "Fastlink: An efficient initial access protocol for millimeter wave systems," in Proc. of the 21st ACM MSWiM Conference, Montreal, CA, Oct. 2018, pp. 109-117.

[9] T. Bai, A. Alkhateeb, and R. W. Heath, "Coverage and capacity of millimeter-wave cellular networks," IEEE Commun. Mag., vol. 52, no. 9, pp. 70-77, 2014.

[10] B. Han, L. Wang, and H. D. Schotten, "A 3D human body blockage model for outdoor millimeter-wave cellular communication," Physical Communication, vol. 25, pp. 502-510, Dec. 2017.

[11] M. Gapeyenko, A. Samuylov, M. Gerasimenko, D. Moltchanov, S. Singh, M. R. Akdeniz, E. Aryafar, N. Himayat, S. Andreev, and Y. Koucheryavy, "On the temporal effects of mobile blockers in urban millimeter-wave cellular scenarios," IEEE Transactions on Vehicular Technology, vol. 66, no. 11, pp. 10124-10138, Nov. 2017.

[12] A. Zhou, L. Wu, S. Xu, H. Ma, T. Wei, and X. Zhang, "Following the shadow: Agile 3-D beam-steering for $60 \mathrm{GHz}$ wireless networks," in Proc. of the IEEE INFOCOM, Honolulu, HI, Apr. 2018.

[13] M. Hashemi, C. E. Koksal, and N. B. Shroff, "Out-of-band millimeter wave beamforming and communications to achieve low latency and high energy efficiency in 5G systems," IEEE Trans. Commun., vol. 66, no. 2, pp. 875-888, 2018.

[14] M. Giordani, M. Mezzavilla, C. N. Barati, S. Rangan, and M. Zorzi, "Comparative analysis of initial access techniques in 5G mmWave cellular networks," in Proc. of the IEEE Annual Conference on Information Science and Systems (CISS), Princeton, NJ, Mar. 2016, pp. 268-273.

[15] M. Giordani, M. Polese, A. Roy, D. Castor, and M. Zorzi, "A tutorial on beam management for 3GPP NR at mmWave frequencies," arXiv preprint arXiv:1804.01908, 2018.

[16] O. Abari, H. Hassanieh, M. Rodriguez, and D. Katabi, "Millimeter wave communications: From point-to-point links to agile network connections," in Proc. of the 15th ACM Workshop on Hot Topics in Networks, Atlanta, GA, Nov. 2016, pp. 169-175.

[17] Q. Xue, X. Fang, and C.-X. Wang, "Beamspace SU-MIMO for future millimeter wave wireless communications," IEEE Journal on Selected Areas in Communications, vol. 35, no. 7, pp. 1564-1575, 2017.

[18] J. Stuckman and G.-Q. Zhang, "Mastermind is np-complete," arXiv preprint cs/0512049, 2005.

[19] 3GPP, "Study on new radio access technology physical layer aspects," 3GPP, Tech. Rep. TR 38.802 v14.2.0, Sep. 2017.

[20] M. Di Renzo, "Stochastic geometry modeling and analysis of multitier millimeter wave cellular networks," IEEE Trans. Wireless Commun., vol. 14, no. 9, pp. 5038-5057, Sep. 2015.

[21] T. Bai and R. W. Heath, "Coverage and rate analysis for millimeter-wave cellular networks," IEEE Trans. Wireless Commun., vol. 14, no. 2, pp. 1100-1114, Feb. 2015

[22] M. K. Samimi and T. S. Rappaport, "3-D millimeter-wave statistical channel model for 5G wireless system design," IEEE Trans. Microw. Theory Tech., vol. 64, no. 7, pp. 2207-2225, 2016.

[23] 3GPP, "Study on channel model for frequency spectrum above $6 \mathrm{GHz}$," 3GPP, Tech. Rep. TR 38.900 v14.3.1, July 2017.

[24] S. Hara and R. Prasad, "Overview of multicarrier CDMA," IEEE Commun. Mag., vol. 35, no. 12, pp. 126-133, 1997.

[25] M. E. Rasekh, Z. Marzi, Y. Zhu, U. Madhow, and H. Zheng, "Noncoherent mmWave path tracking," in Proc. of the 18th ACM International Workshop on Mobile Computing Systems and Applications, Sonoma, CA, Feb. 2017, pp. 13-18 